\title{
SIMPLIFIED MODEL OF THE HEAT EXCHANGE PROCESS IN ROTARY REGENERATIVE AIR PRE-HEATER ${ }^{1}$
}

\author{
Abdulla A. Azamov \\ Institute of Mathematics, National University of Uzbekistan, \\ Tashkent, Uzbekistan, \\ abdulla.azamov@gmail.com \\ Mansur A. Bekimov \\ Institute of Mathematics, National University of Uzbekistan, \\ Tashkent, Uzbekistan, \\ mansu@mail.ru
}

\begin{abstract}
A simplified mathematical model of a rotary regenerative air pre-heater (RRAP) is suggested and studied based on the averaged dynamics of the heat exchange process between nozzles and a heat carrier (i.e. air or gas-smoke mixture). Averaging in both spatial coordinates and time gives a linear discrete system that allows deriving explicit formulas for determining the characteristics of the air heater and establishing some properties such as periodicity, stability, ergodicity and others.
\end{abstract}

Key words: Heat exchange, Cyclic process, Averaging, Linear discrete system, Stability, Ergodicity, Inverse problem.

\section{Introduction}

A rotary regenerative air pre-heater (RRAP) is a special unit, usually applied in thermal power plants (TPP) in order to increase its efficiency by heating the air which is blowing into a boiler of the plant by means of an exhaust of hot mixture of smoke and gas (from now on simply gas) generated in fuel combustion. The RRAP essentially reduces the thermal pollution of atmosphere [6], [8-12].

Currently, several types of RRAPs are used in TPP. In the present paper, we consider the case of a unit with the main part consisting of a rotating cylindrical drum with metal nozzles of high thermal conductivity. The region of space occupied by the wheel of the RRAP is divided into two parts $B_{A}$ and $B_{G}$ by a fixed conditional plane passing through the axis of the cylinder. During the work of the RRAP, the atmospheric air passes through the part $B_{A}$ in one direction parallel to the axis of the drum, being heated by the nozzles; as a result, the temperature of the nozzles is reduced. Through the part $B_{G}$, the gas flows in the opposite direction, being cooled by the heat output to the nozzles. The final transfer of heat from the hot gas to the cool air occurs due to the rotation of the drum around its axis.

Monitoring and control of the temperatures of air and gas leaving the RRAP and especially the temperature of the nozzles is the important problem for the effective exploitation of the RRAP [3], [15], [17-19]. The direct measurement of the temperatures of air and gas of both inlet and outlet is easily carried out; in contrast, the observation and control of the nozzle temperature require to use sophisticated measuring tools and represent an important problem. Therefore, the treatment for mathematical modeling of the heat exchange process in the RRAP is reasonable.

\footnotetext{
${ }^{1}$ This work was supported by Committee for Coordination Science and Technology Development Under Cabinet of Ministers of Uzbekistan (project no F4-FA-F014).
} 
In recent decades, a variety of mathematical models of the heat exchange process in RRAPs were suggested [1-3], [5] and see also [8]. It should be noted that the thermodynamic process in RRAP nozzles can be in principle described by the classical heat conduction equation, but, without additional simplifying assumptions, it is difficult to write out the boundary conditions since the nozzle system has a rather complex geometry. On the other hand, modeling of the thermodynamic process of air heating and gas cooling passing through the drum of the RRAP is even more challenging problem pertaining to thermos-aerodynamics [8]. Furthermore, the necessity of considering the turbulence of flow that arises when air and gas pass through the RRAP drum also adds complexity. Because of these and other features, all mathematical models of the RRAP are built under essential simplifying assumptions.

In the present paper, we propose a mathematical model of the thermodynamic process in the RRAP based on averaging the quantities associated with the heat exchange process between the nozzles, air and gas in both spatial coordinates and time interval. As a result, we obtain rather simple linear discrete equation. This allows us to write out explicit calculating formulas for the current values of parameters, and find steady and periodical states, establish the ergodicity and other properties. Then, we provide appropriate calculations for the case when averaging over the time variable is carried out on a period of time equal to a half-cycle of rotation of the RRAP drum. In subsequent parts of the work, models with averaging performed over a small time interval are considered, a comparative analysis of numerical results and experimental data is fulfilled.

\section{Derivation of equations}

Let the RRAP drum be of the form of a cylinder $x^{2}+y^{2} \leq R^{2}, 0 \leq z \leq H$. Assume that the parts $B_{A}$ and $B_{G}$ are described by conditions $y \geq 0$ and $y \leq 0$, respectively. Let $\Theta(t, x, y, z)$ denote the temperature at the point $(x, y, z)$ of the drum occupied by the nozzles at time $t, t \geq 0$, and let $T(t, x, y, z)$ denote the temperature of heat transfer (air or gas) at the point $(x, y, z)$ of the drum outside the nozzles. The pair of quantities $\Theta(t, x, y, z)$ and $T(t, x, y, z)$ completely characterizes the heat change process in the RRAP drum. However, as mentioned above, due to the complexity of the configuration of the nozzles, the initial-boundary problem for corresponding system of equations of thermo-aerodynamics is too hard to be investigated by analytical methods. One of the ways to overcome such complexity is the method of averaging [4], [7]. To this end, we consider the corresponding average values

$$
\begin{aligned}
& \Theta_{A}(t)=\frac{1}{V\left(B_{A}^{\bullet}\right)} \int_{B_{\dot{A}}^{\bullet}} \Theta_{A}(t, x, y, z) d v, \quad \Theta_{G}(t)=\frac{1}{V\left(B_{G}^{\bullet}\right)} \int_{B_{G}^{\bullet}} \Theta_{G}(t, x, y, z) d v, \\
& T_{A}(t)=\frac{1}{V\left(B_{A}^{\circ}\right)} \int_{B_{A}^{\circ}} T_{A}(t, x, y, z) d v, \quad T_{G}(t)=\frac{1}{V\left(B_{G}^{\circ}\right)} \int_{B_{G}^{\circ}} T_{G}(t, x, y, z) d v,
\end{aligned}
$$

where $B_{A}^{\bullet}=B_{A} \cap \bar{B}, B_{G}^{\bullet}=B_{A} \cap \bar{B}, \bar{B}$ is the part of the drum occupied by the nozzles, $B_{A}^{\circ}=$ $B_{A} \backslash B_{A}^{\bullet}, B_{G}^{\circ}=B_{G} \backslash B_{G}^{\bullet}, d v$ is the volume element, $V$ denotes the volume of the corresponding part of the drum.

Next, we perform averaging over the time intervals $I(n)=[n h,(n+1) h)$ as well, where $h$ is the half-turn time of the RRAP drum, $n=0,1,2, \ldots$. Denote the average temperatures obtained in this way by

$$
\begin{aligned}
x_{n} & =\frac{1}{h} \int_{I(n)} \Theta_{A}(t) d t, & y_{n} & =\frac{1}{h} \int_{I(n)} \Theta_{G}(t) d t, \\
u_{n} & =\frac{1}{h} \int_{I(n)} T_{A}(t) d t, & v_{n} & =\frac{1}{h} \int_{I(n)} T_{G}(t) d t .
\end{aligned}
$$

Equations connecting these quantities are derived under the following simplifying assumptions: 
1. for $n h \leq t<(n+1) h$, the RRAP drum is motionless, the portion of air (respectively, gas) filling the part $B_{A}^{\mathrm{o}}$ (respectively, $B_{G}^{\mathrm{o}}$ ) is also motionless, the heat exchange between the heat carrier and nozzles occurs in accordance with the linear Newton law [8];

2. at the time $t=(n+1) h$, the heated portion of air with temperature $u_{n}$ leaves the area $B_{A}^{\mathrm{o}}$, the gas portion cooled down to temperature $v_{n}$ leaves the region $B_{G}^{\mathrm{o}}$, the drum turns to $180^{\circ}$ with jump (i.e., the part $B_{A}^{\bullet}$ goes to the part $B_{G}^{\bullet}$ and vice versa); then the part $B_{A}^{\text {o }}$ is filled in by a new portion of air from outward (or from calorifer in case of its connection to the RRAP, [12]) of temperature $p_{n}$, and the part $B_{G}^{o}$ is filled in by a new portion of gas of temperature $q_{n}$.

In accordance with the Newton law, we have the following relations:

$$
\begin{gathered}
x_{n+1}=(1-\beta h) y_{n}+\beta h q_{n}, \quad y_{n+1}=(1-\alpha h) x_{n}+\alpha h p_{n}, \\
u_{n}=p_{n}+\gamma h\left(x_{n}-p_{n}\right), \quad v_{n}=q_{n}+\delta h\left(y_{n}-q_{n}\right),
\end{gathered}
$$

where $\alpha, \beta, \gamma, \delta$ are parameters depending on characteristics of the heat exchange process in the RRAP (the geometry, the heat capacity of the carcass of drum, the system of nozzles and their structure, the humidity of air and gas and their thermodynamic characteristics, the coefficients of heat conductivity and diffusion, parameters that characterize the heat exchange process on the contact surface of the nozzles with air and gas and etc.).

Relations (1.1) and (1.2) have been obtained as a result of extremely simplifying assumptions on the heat exchange process in the RRAP. Nevertheless, due to such simplification, system (1.1) allows a fairly complete analysis; therefore, it can serve as a basic model to describe the work of the RRAP.

\section{Solution of the system}

Introducing the vectors $z_{n}=\left(x_{n}, y_{n}\right)^{T}$ and $r_{n}=h\left(\beta q_{n}, \alpha p_{n}\right)^{T}$, where $T$ is the transpose sign to transform the row-vector to the column-vector, and the matrix

$$
A=\left(\begin{array}{cc}
0 & 1-\beta h \\
1-\alpha h & 0
\end{array}\right)
$$

one can rewrite system (1.1) as follows:

$$
z_{n+1}=A z_{n}+r_{n}, \quad n=0,1,2, \ldots
$$

All further reasoning is conducted under the assumption $0<\alpha h, \beta h<1$, called the physical realizability of the model. This condition implies the fact that the eigenvalues of the matrix $A$, which are $\pm \mu$ where $\mu=\sqrt{(1-\alpha h)(1-\beta h)}$, belong to the interval $(-1,1)$ and therefore all the solutions of system (1.1) are asymptotically stable [13,14], [16].

The solution of equation (2.1) can be written by the Cauchy formula [13]

$$
z_{n}=A^{n} z_{0}+\sum_{k=0}^{n-1} A^{n-1-k} r_{k} .
$$

Since $A^{n}=\mu^{n} E$ for even numbers $n$, and $A^{n}=\mu^{n-1} A$ for odd numbers $n$, then equation (2.2) can be transformed to the form

$$
z_{n}=\mu^{n} z_{0}+A \sum_{j=0}^{n / 2-1} \mu^{n-2-2 j} r_{2 j}+\sum_{j=0}^{n / 2-1} \mu^{n-2-2 j} r_{2 j+1},
$$


for even $n$, and to the form

$$
z_{n}=\mu^{n-1} A z_{0}+A \sum_{j=1}^{(n-1) / 2} \mu^{n-1-2 j} r_{2 j-1}+\sum_{j=0}^{(n-1) / 2} \mu^{n-1-2 j} r_{2 j}
$$

for odd $n$.

Based on these relations, one can easily obtain explicit formulas for $x_{n}$ and $y_{n}$. In practice, however, it is more convenient to calculate them, in case of need, directly from equations (1.1) that are accommodated to computer calculations. In the rest of the paper, some properties of solution of systems (1.1), (1.2) are established.

\section{Steady state and periodic regimes}

Let us first consider the case of a steady state, which occurs under the assumption of constant incoming flows. Such a state can be established in the periods of time measured in hours, if the parameters of the air and the energy load at the TPP remain virtually unchanged. Since $h$ is measured in minutes, the work of the RRAP actually consists of long periods of steady states and relatively short transition intervals from one steady state to another. Therefore, it is important to determine the parameters of the RRAP under steady state conditions.

Thus, let

$$
p_{n} \equiv \bar{p}, \quad q_{n} \equiv \bar{q}, \quad n=0,1,2, \ldots, \quad \bar{r}=(\beta \bar{q}, \quad \alpha \bar{p})^{T} .
$$

Then formula (2.2) can be simplified even more: for even $n$

$$
z_{n}=\mu^{n} z_{0}+\left(1-\mu^{n}\right)(E-A)^{-1} \bar{r}
$$

or in the component form

$$
\begin{aligned}
& x_{n}=\mu^{n} x_{0}+\frac{1-\mu^{n}}{\alpha+\beta-\alpha \beta h}[p+q(1-\beta h)], \\
& y_{n}=\mu^{n} y_{0}+\frac{1-\mu^{n}}{\alpha+\beta-\alpha \beta h}[q+p(1-\alpha h)],
\end{aligned}
$$

and for odd $n$

$$
z_{n}=\mu^{n-1} A z_{0}+\frac{1}{\alpha+\beta-\alpha \beta h}\left(E-\mu^{n} A\right)(E-A)^{-1} r
$$

or

$$
\begin{aligned}
& x_{n}=\mu^{n-1}(1-\beta h) y_{0}+\frac{1}{\alpha+\beta-\alpha \beta h}\left[p\left(1-\mu^{n+2}\right)+q(1-\beta h)\left(1-\mu^{n-1}\right)\right], \\
& y_{n}=\mu^{n-1}(1-\alpha h) x_{0}+\frac{1}{\alpha+\beta-\alpha \beta h}\left[q\left(1-\mu^{n+2}\right)+p(1-\alpha h)\left(1-\mu^{n-1}\right)\right] .
\end{aligned}
$$

For the steady state, we obtain $\bar{z}=(E-A)^{-1} \bar{r}$ from the equation $\bar{z}=A \bar{z}+\bar{r}$ (the invertibility of the matrix $E-A$ follows from the condition of physical realizability) or in the component form

$$
\begin{aligned}
& \bar{x}=\frac{1}{\alpha+\beta-\alpha \beta h}(\alpha p+\beta q(1-\beta h)), \\
& \bar{y}=\frac{1}{\alpha+\beta-\alpha \beta h}(\beta q+\alpha p(1-\alpha h)) .
\end{aligned}
$$

Due to the fact noticed above, steady state (3.2) is asymptotically stable. On time intervals measured in weeks, a periodic state for the RRAP can be formed. 
Theorem 1. Let the sequence $r_{n}$ be periodic with the period $m, m \geq 2$, i.e. $r_{n+m}=r_{n}$, where $n=n_{0}, n_{0}+1, n_{0}+2, \ldots ; n_{0}$ is some fixed value of $n$. Then system (2.1) has a unique periodic solution of period $m$, which is asymptotically stable.

P r o o f. The uniqueness and asymptotic stability follows from the condition of physical realizability. To establish the existence, we set

$$
z_{n}^{*}=\left(E-A^{m}\right)^{-1} \sum_{k=0}^{m-1} A^{m-1-k} r_{k}
$$

(it follows from the condition of physical realizability that the matrix $E-A^{m}$ is invertible).

Let $z_{n}^{*}$ be a trajectory with the initial point $z_{0}^{*}$. Rewrite relation (3.3) as follows:

$$
z_{n}^{*}=A^{m} z_{0}^{*}+\sum_{k=0}^{m-1} A^{m-1-k} r_{k}=z_{m}^{*} .
$$

Then,

$$
z_{n+m}^{*}=A^{n+m} z_{0}^{*}+\sum_{k=0}^{m-1} A^{n+m-1-k} r_{k}+\sum_{k=m}^{n+m-1} A^{n+m-1-k} r_{k} .
$$

The sum of the first two summands in the right hand side of (3.4) is $A^{n} z_{m}^{*}$, the third one is equal to

$$
\sum_{k=0}^{n-1} A^{n-1-k} r_{k+m}=\sum_{k=0}^{n-1} A^{n-1-k} r_{k}
$$

since $r_{k}$ is periodic. Thus,

$$
\sum_{k=0}^{n-1} A^{n-1-k} r_{k}=z_{n}-A^{n} z_{0} .
$$

Now, in accordance with (3.4), we obtain

$$
z_{n+m}^{*}=A^{n} z_{m}^{*}+z_{n}-A^{n} z_{0}^{*}=z_{n},
$$

and the proof is complete.

\section{Boundedness and ergodicity of the solutions}

In time intervals of longer duration, in a changeable external environment and energy load at the TPP, the sequence $r_{n}$ is not periodic, a fortiori, it is not stationary. In this regard, we give two properties of the solution for more general classes of systems (2.1), which models more or less irregular regime of the heat exchange process.

Theorem 2. If $r_{n}$ is a bounded sequence, then each solution of equation (2.1) is also bounded.

This statement is also a special case of more general theorem [11].

Theorem 3. Let $\lim _{n \rightarrow \infty} r_{n}=l$. Then each solution $z_{n}$ approaches the limit $(E-A)^{-1} l$ as $n \rightarrow \infty$ independently of $z_{0}$. 
P r o o f. First assume that $l=0$. Let $M=\max _{n}\left|r_{n}\right|$. Given any $\varepsilon>0$, choose $n_{0}(\varepsilon)$ such that $\left|r_{n}\right|<\frac{1}{2}(1-\|A\|) \varepsilon$ at $n \geq n_{0}(\varepsilon)$. Then choose $N\left(n_{0}(\varepsilon)\right)$ such that

$$
\mu^{n}<\frac{1-\|A\|}{2 M\left(1-\left\|A^{n_{0}}\right\|\right)} \varepsilon
$$

for all $n \geq N\left(n_{0}(\varepsilon)\right)$. Clearly, $N\left(n_{0}(\varepsilon)\right)$ can be chosen to satisfy $N\left(n_{0}(\varepsilon)\right) \geq n_{0}(\varepsilon)$. Then, for $n \geq N\left(n_{0}(\varepsilon)\right)$, we have

$$
\left|z_{n}\right|<\left\|A^{n}\right\|\left|z_{0}\right|+\left|\sum_{k=0}^{n_{0}-1} A^{n-1-k} r_{k}\right|+\left|\sum_{k=n_{0}}^{n-1} A^{n-1-k} r_{k}\right| .
$$

Since $0<\alpha h, \beta h<1$, the first summand tends to zero as $n \rightarrow \infty$. Denote two other terms by $S_{n}^{I}$ and $S_{n}^{I I}$. Then, in view of $\|A\|=\mu \in(0,1)$, we get

$$
\begin{gathered}
S_{n}^{I}<\left\|A^{n-n_{0}}\right\| \sum_{k=0}^{n_{0}-1}\left\|A^{n_{0}-1-k}\right\|\left|r_{k}\right|<M\left\|A^{n-n_{0}}\right\| \sum_{k=0}^{n_{0}-1}\left\|A^{n_{0}-1-k}\right\|<M \mu^{n} \frac{1-\left\|A^{n_{0}}\right\|}{1-\|A\|}<\frac{\varepsilon}{2}, \\
S_{n}^{I I}<\sum_{k=n_{0}}^{n-1}\left\|A^{n-1-k}\right\|\left|r_{k}\right|<\frac{1-\|A\|}{2} \varepsilon \sum_{k=0}^{\infty}\left\|A^{k}\right\|=\frac{1-\|A\|}{2} \varepsilon \frac{1}{1-\|A\|}<\frac{\varepsilon}{2} .
\end{gathered}
$$

Hence, $\left|z_{n}\right| \leq S_{n}^{I}+S_{n}^{I I}<\varepsilon$ at $n \geq N\left(n_{0}(\varepsilon)\right)$, i.e. $z_{n} \rightarrow 0$.

Let now $r_{n} \rightarrow l$ as $n \rightarrow \infty$, where $l \neq 0$. We make the change of variables

$$
z_{n}=\bar{z}_{n}+(E-A)^{-1} l, \quad \bar{r}_{n}=r_{n}-l .
$$

Then

$$
\bar{z}_{n+1}+(E-A)^{-1} l=A\left[\bar{z}_{n}+(E-A)^{-1} l\right]+\bar{r}_{n}+l=A \bar{z}_{n}+\bar{r}_{n},
$$

where $\bar{r}_{n} \rightarrow 0$. As proved, $\bar{z}_{n} \rightarrow 0$. Therefore, $z_{n} \rightarrow(E-A)^{-1} l$.

In general, the change of the parameters characterizing the state of air, as well as the variation of the load at the TPP is random with a hard-determinable distribution function.

Considering the work of the RRAP as a stochastic process, we leave it for the next part of the paper, and we now present another property, taking into account irregular characters of values of phase variables of system (1.1), (1.2).

A sequence $a_{n}$ is called almost-periodic, if it can be represented in the form $b_{n}+c_{n}$, where $b_{n}$ is periodic and $c_{n} \rightarrow 0$ as $n \rightarrow \infty$.

Corollary 1. If $r_{n}$ is almost-periodic, then each solution of (2.2) is also almost-periodic.

Definition. A sequence $x_{n}$ is called ergodic, if the sequence of Cesaro means

$$
\sigma_{n}=\frac{x_{1}+x_{2}+\cdots+x_{n}}{n}
$$

converges as $n \rightarrow \infty$.

Theorem 4. If the sequence $r_{n}$ is ergodic, namely

$$
\rho_{n}=\frac{r_{0}+r_{1}+\cdots+r_{n-1}}{n} \rightarrow l
$$

then each solution $z_{n}$ is also ergodic with

$$
S_{n}=\frac{z_{1}+z_{2}+\cdots+z_{n}}{n} \rightarrow(E-A)^{-1} l .
$$


P r o o f. Express $S_{N}$ as the sum of two terms: $S_{N}=\Sigma_{I}+\Sigma_{I I}$, where

$$
\Sigma_{I}=\frac{1}{N} \sum_{n=1}^{N} A^{n} z_{0}, \quad \Sigma_{I I}=\frac{1}{N} \sum_{n=1}^{N} \sum_{k=0}^{n-1} A^{n-1-k} r_{k} .
$$

Then

$$
\Sigma_{I}=\frac{1}{N} \sum_{n=1}^{N} A^{n} z_{0}=\frac{1}{N} A(E-A)^{-1}\left(E-A^{N}\right) z_{0} \rightarrow 0
$$

as $n \rightarrow \infty$.

For $\Sigma_{I I}$, we have

$$
\begin{aligned}
\Sigma_{I I}= & \frac{1}{N} \sum_{k=0}^{N-1} \sum_{n=k+1}^{N} A^{n-1-k} r_{k}=\frac{1}{N} \sum_{k=0}^{N-1}\left(\sum_{n=0}^{N-1-k} A^{n}\right) r_{k} \\
& =\frac{(E-A)^{-1}}{N}\left[\sum_{k=0}^{N-1} r_{k}-\sum_{k=0}^{N-1} A^{N-1-k} r_{k}\right] \\
& =(E-A)^{-1} \rho_{n}-\frac{(E-A)^{-1}}{N} \sum_{k=0}^{N-1} A^{N-1-k} r_{k} .
\end{aligned}
$$

By hypothesis of the theorem, $(E-A)^{-1} \rho_{n} \rightarrow(E-A)^{-1} l$ as $N \rightarrow \infty$. Assuming $\rho_{k}=\xi_{k}+l$, where $\xi_{k} \rightarrow 0$, we arrive at the equation

$$
r_{k}=(k+1) \rho_{k+1}-k \rho_{k}=(k+1) \xi_{k+1}-k \xi_{k}+l .
$$

Thus,

$$
\frac{(E-A)^{-1}}{N} \sum_{k=0}^{N-1} A^{N-1-k} r_{k}=\frac{(E-A)^{-1}}{N}\left[\sum_{k=0}^{N-1} A^{N-1-k}\left[(k+1) \xi_{k+1}-k \xi_{k}\right]-\sum_{k=0}^{N-1} A^{N-1-k} l\right] .
$$

Obviously,

$$
\sum_{k=0}^{N-1} A^{N-1-k} l \rightarrow 0
$$

as $N \rightarrow \infty$. Let

$$
S=\frac{1}{N} \sum_{k=0}^{N-1} A^{N-k-1} k \xi_{k} .
$$

Show that $S \rightarrow 0$ as $N \rightarrow \infty$. Indeed,

$$
|S| \leq \sum_{k=0}^{N-1} \mu^{N-1-k} \frac{k}{N}\left|\xi_{k}\right| \leq \sum_{k=0}^{N-1} \mu^{N-1-k}\left|\xi_{k}\right| .
$$

By Theorem 3, the right-hand side of this inequality tends to 0 as $N \rightarrow \infty$. Thus, finally we obtain

$$
S_{N}=\frac{1}{N} \sum_{n=1}^{N} z_{n} \rightarrow(E-A)^{-1} l,
$$

which is our claim. 


\section{Finding the coefficients of the system}

As mentioned above, besides the temperature and velocity of incoming air and gas, the drum rotation speed, the heat exchange process in the RRAP depends on many parameters expressing the thermodynamic characteristics of air and gas, the material and geometry of the nozzles of the RRAP, thermal properties of the drum casing etc.

There are a number of works devoted to the mathematical modeling of the RRAP where formulas are given to calculate the values of the parameters above on the basis of molecular physics laws [1-3], [8], [9]. In addition, finding the numerical parameters characterizing the RRAP is only possible with a certain accuracy. From this point of view, it is much easier to define them with a satisfactory accuracy by solving the inverse problem for system (1.1), (1.2) on the basis of empirical data. With regard to model (1.1), (1.2), such a problem consists in calculating the values of $\alpha, \beta, \gamma$, and $\delta$ based on the results of measurements of temperatures of outgoing air $u_{n}$ and gas $v_{n}$. Here, there is a wide field of application of the least squares method and tools of mathematical statistics. Here, we confine ourselves to the simplest case, when $\alpha, \beta, \gamma$, and $\delta$ are found by measuring $u_{1}, u_{2}, v_{1}$, and $v_{2}$ assuming that incoming streams $p_{n}=p, q_{n}=q$ are stationary. We can assume that $u_{0}=p, v_{0}=q$, and the values of $u_{1}, u_{2}, v_{1}$, and $v_{2}$ are found by the direct measurements. As a result, we arrive at the problem of finding the unknowns $\alpha, \beta, \gamma, \delta$ based on the given values $h, p, q, u_{1}, u_{2}, v_{1}, v_{2}$, without measuring the values $x_{n}, y_{n}$ (the nozzle temperature).

We have

$$
\begin{array}{cl}
x_{2}=(1-\beta h) y_{1}+\beta h q, & y_{2}=(1-\alpha h) x_{1}+\alpha h p, \\
u_{1}=p+\gamma h\left(x_{1}-p\right), & v_{1}=q+\delta h\left(y_{1}-q\right), \\
u_{2}=p+\gamma h\left(x_{2}-p\right), & v_{2}=q+\delta h\left(y_{2}-q\right) .
\end{array}
$$

Set $\bar{u}_{k}=u_{k}-p, \quad \bar{v}_{k}=v_{k}-q, k=1,2$, (these values have the clear physical meaning). Substituting the values of $x_{2}$ and $y_{2}$ from (5.1) into (5.3), we obtain the following system

$$
\begin{gathered}
\gamma h\left(x_{1}-p\right)=\bar{u}_{1}, \quad \delta h\left(y_{1}-q\right)=\bar{v}_{1}, \\
\gamma h\left[(1-\beta h) y_{1}+\beta h q-p\right]=\bar{u}_{2}, \quad \delta h\left[(1-\alpha h) x_{1}+\alpha h p-q\right]=\bar{v}_{2},
\end{gathered}
$$

with 6 unknowns $\alpha, \beta, \gamma, \delta, x_{1}, y_{1}$. It is nonlinear and, in general, cannot be solved explicitly.

Therefore, we use the fact that, for the values of $\alpha, \beta, \gamma, \delta$, there is a priori estimate $0.1 \div 0.6$ and $h<1$. This allows us to neglect the terms containing $\beta \gamma h^{2}$ and $\alpha \delta h^{2}$.

Then equations (5.5) take the form $\gamma h\left(y_{1}-p\right)=\bar{u}_{2}, \delta h\left(x_{1}-q\right)=\bar{v}_{2}$. As a result, for the intermediate unknowns $x_{1}, y_{1}$, we obtain the linear system

$$
\frac{x_{1}-p}{y_{1}-p}=\frac{\bar{u}_{1}}{\bar{u}_{2}}, \quad \frac{y_{1}-q}{x_{1}-q}=\frac{\bar{v}_{2}}{\bar{v}_{1}}
$$

with the determinant equal to $\bar{u}_{1} \bar{v}_{1}-\bar{u}_{2} \bar{v}_{2}$. We call the quantity $\chi=\left|\bar{u}_{1} \bar{v}_{1}-\bar{u}_{2} \bar{v}_{2}\right|$ the divergence coefficient of the RRAP. The deviation of $\chi$ from zero is a characteristic of the RRAP that expresses how the rates of air heating and gas cooling differ. Further, we assume $\chi \neq 0$. It follows from (5.6) that

$$
x_{1}=\frac{\left(\bar{u}_{1}-\bar{u}_{2}\right) \bar{v}_{2} p+\left(\bar{v}_{1}-\bar{v}_{2}\right) \bar{u}_{1} q}{\bar{u}_{1} \bar{v}_{1}-\bar{u}_{2} \bar{v}_{2}}, \quad y_{1}=\frac{\left(\bar{u}_{1}-\bar{u}_{2}\right) \bar{v}_{1} p+\left(\bar{v}_{1}-\bar{v}_{2}\right) \bar{u}_{2} q}{\bar{u}_{1} \bar{v}_{1}-\bar{u}_{2} \bar{v}_{2}} .
$$

Substituting the values $x_{1}, y_{1}$ into (5.4), (5.5), we obtain the final formulas

$$
\alpha h=\frac{x_{1}-q}{x_{1}-p} \bar{v}_{1}-\frac{y_{1}-q}{x_{1}-p} \bar{v}_{2}, \quad \beta h=\frac{y_{1}-p}{y_{1}-q} \bar{u}_{1}-\frac{x_{1}-p}{y_{1}-q} \bar{u}_{2}
$$




$$
\gamma h=\frac{\bar{u}_{1}}{x_{1}-p}, \quad \delta h=\frac{\bar{v}_{1}}{y_{1}-q} .
$$

Table 1 shows posteriori values of parameters $\alpha, \beta, \gamma, \delta$ calculated by these formulas under the assumption that $p=32^{\circ}, q=282^{\circ}, h=0.25[4,5]$.

\begin{tabular}{|c|c|c|c|c|c|c|c|}
\hline $\bar{u}_{1}$ & $\bar{v}_{1}$ & $\bar{u}_{2}$ & $\bar{v}_{1}$ & $\alpha$ & $\beta$ & $\gamma$ & $\delta$ \\
\hline 3.61 & -2.58 & 6.75 & -4.82 & 0.048 & 0.068 & 0.041 & 0.029 \\
\hline
\end{tabular}

Table 1. Posteriori values of parameters $\alpha, \beta, \gamma, \delta$.

\section{Conclusion}

In the present paper, we have proposed the mathematical model of thermodynamic process of the RRAP, which is described by linear discrete equations. To obtain this, we have used averaging the quantities associated with the heat exchange process between the nozzles, air and gas in both the spatial coordinates and time interval. We have found steady and periodical states, established the ergodicity and other properties. Next, we have studied the cases when the time averaging is performed over the period of time equal to the half-cycle of rotation of the RRAP drum as well as when the time averaging is performed over a small time interval. Finally, we have provided the comparative analysis of the numerical results obtained and the experimental data.

\section{Acknowledgements}

Authors express their gratitude to G.I. Ibragimov for useful discussion and help.

\section{REFERENCES}

1. Alagic S., Kovacevic A. and Buljubasic I. A numerical analysis of heat transfer and fluid flow in rotary regenerative air pre-heaters // Strojniški Vestnik, 2005. Vol. 51, no. 7-8, P. 411-417.

2. Armin Heidari-Kaydan, Ebrahim Hajidavalloo. Three-dimensional simulation of rotary air preheater in steam power plant // Applied Thermal Engineering, 2014. Vol. 73. P. 397-405.

3. Boštjan Drobnič, Janez Oman, Matija Tuma. A numerical model for the analysis of heat transfer and leakages in a rotary air preheater //Int. J. of Heat and Mass Transfer, 2006. Vol. 49. P. 5001-5009.

4. Burd V.Sh. Method of averaging for differential equations on an infinite interval: Theory and Applications. Chapman and Hall/CRC, 2007. 360 p.

5. Chi-Liang Lee. Regenerative air preheaters with four channels in a power plant system // J. of Chinese Institute of Engineers. 2009. Vol. 32, no. 5, P. 703-710.

6. Grebennikov E.A. Method of averaging in applied problems. Moscow: Nauka, 1986. 256 p. [in Russian]

7. Kirsanov Yu.A. Cyclic thermal processes and the theory of thermal conductivity in regenerative air heaters. Moscow: Fizmatlit, 2007. 240 p. [in Russian]

8. Kovalevskii V.P. Simulation of heat and aerodynamic processes in regenerators of continuous and periodic operation. I. Nonlinear mathematical model and numerical algorithm // J. of Engineering Physics and Thermophysics, 2004. Vol. 77, no. 6, P. 1096-1109.

9. Kudinov A.A. The study of heat transfer processes in rotary regenerative air power boilers // J. of Energetics, 2012. No 6. P. 32-34. [in Russian]

10. Kudinov A.A., Ziganshina S.K. Energy savings in power and heat technologies. Moscow: Mashinostroenie, 2011. 374 p. [in Russian]

11. Liu Fuguo, Zhou Xingang. Heat transfer model of tri-section rotary air preheater and experimental verification // J. of Mechanical Engineering, 2010. Vol. 46, no 22, P. 144-150. 
12. Ismatkhodjayev S.K., Nuraliyev E.N., Abduraimov F.E., Mokrushev V.A., Azamov A.A., Bekimov M.A. A method for automatic adjusting the air temperature in air heater. Patent No IAP 05072. [in Russian]

13. Romanko V.K. The course of difference equations. oscow: Fizmatlit, 2012. 200 p. [in Russian]

14. Saber Elaydi. An introduction to difference equations. Third edition, Springer Science+Business Media, New York, 2005.

15. Shruti G., Ravinarayan Bhat, Gangadhar Sheri. Performance evaluation and optimization of air preheater in thermal power plant // International J. of Mechanical Engineering and Technology (IJMET), 2014. Vol. 5, iss. 9. P. 22-30.

16. Srochko V.A. Numerical methods: Lectures. Irkutsk: Irkutsk University Press, 2004. 205 p. [in Russian]

17. Wang H.Y. Analysis on thermal stress deformation of rotary air-preheater in a thermal power plant // Korean J. Chem. Eng., 2009. Vol. 26, P. 833-839.

18. Wang H.Y., Zaho L.L., Xu Z.G., Chun W.G., Kim H.T. The study on heat transfer model of tri-sectional rotary air preheater based on the semi-analytical method// Appl. Therm. Eng. 2008. Vol. 28, no. 14-15. P. 1882-1888.

19. Zhuang Wu, Roderick V.N. Melnik, Finn Borup. Model-based analysis and simulation of regenerative heat wheel // Energy and Buildings, 2006. No. 28. P. 502-514. 